\title{
Successive Unfolding of Family Preferences
}

\author{
Joseph Lee Rodgers and Forrest W. Young \\ University of North Carolina at Chapel Hill
}

A technique to scale preferences in relation to an externally derived stimulus configuration, called Successive Unfolding, is described. Four steps are involved: (1) computing a matrix of inter-stimulus distances; (2) using ALSCAL to obtain a stimulus configuration from the matrix of distances; (3) using Carroll's regression procedure to solve for subject ideal points; and (4) using this starting configuration to scale preference rank orders in ALSCAL. The technique is used to analyze family preference data. Results suggest that a number preference, a sex preference, and a balance preference are the components contributing to overall family preferences. Race and sex differences are portrayed by locating subject ideal points along these dimensions. Finally, the relationship of Successive Unfolding to previous techniques for measuring family preferences is discussed, and the decision-making process modeled by Successive Unfolding is outlined.

The analysis of preference data is of great importance to psychological researchers. There are several reasons for this. First, the very idea that individuals have preferences suggests individual differences, an important topic in the psychological literature. Second, the understanding of preferences has obvious economic and social benefits. And third, techniques for collecting preference data, (e.g., paired comparison techniques and rank order techniques) are well

APPLIED PSYCHOLOGICAL MEASUREMENT

Vol. 5, No. 1, Winter 1981, pp. 51-62

(c) Copyright 1981 Applied Psychological Measurement Inc. studied and well defined (see Coombs, 1964; Torgerson, 1958). Given these, methods to analyze preference data are quite important. $A$ popular method for converting preference data to a spatial representation has been multidimensional unfolding analysis. A relatively recent development in this area is external unfolding analysis. In this paper a technique for external unfolding will be described and its use illustrated through application to some family preference data.

\section{Unfolding Analysis}

Coombs (1950) introduced unfolding analysis as a unidimensional scaling technique. The goal of unfolding analysis in its multidimensional extension is the scaling of both subject and stimulus points into a common space. Given a configuration containing both subject and stimulus points, the rank order of the distances from a subject's point to the various stimulus points ideally corresponds to the rank order of the subject's preferences for the various stimuli. Therefore, a subject's point is called his/her "ideal point," and the scaling is successful to the extent that his/her preference rank order is perfectly "unfolded" into the space. Unfolding analysis can be classified into two categories-internal unfolding and external unfolding. 
In internal unfolding the subject and stimulus points are scaled simultaneously. This approach can be conceptualized in the following way. First, a stimulus point is placed arbitrarily into an $\boldsymbol{n}$-dimensional space. All the subject points are scaled into the space according to the subjects' preferences for that stimulus. Next, another stimulus point is placed into the space, and the subject points are adjusted according to preferences for this stimulus, while maintaining the appropriate relationships between the subject points and the first point (which itself may be adjusted for this purpose). This process continues, and as each stimulus point is placed in the space, appropriate adjustments are made in the location of the subject points and the previous stimulus points. In the early stages of this process, there is a great deal of freedom for placing subject points in the space while maintaining the preference rank orders. That is, a subject point may fall anywhere in a large area of the space and still be an "ideal point." As more and more stimulus points are placed in the space, this freedom is decreased. Finally, it may become impossible to find "ideal points" for every subject. This problem may be solved by using a higher dimensionality or by maximizing a "fit" criterion so that the solution is as good as possible (though not perfect).

Relationships may exist among stimuli placing constraints on the adjustments allowed in the stimulus points. The researcher may, for instance, have a predetermined or theoretically defined stimulus configuration, and he/she may wish to scale subject points in relation to this configuration. In this case the conceptualization presented above must be revised. The structural relationships between the stimuli already exist, and these relationships are nonadjustable in the scaling. What remains is to locate the subject's ideal points, again so that the rank order of distances to stimulus points reflects the rank order of his/her preferences. This is the external unfolding problem proposed by Carroll (1972) to relate "preference data to a given stimulus space."
The solutions to this and other unfolding problems are not really derived as described above. Rather, analytical procedures or iterative algorithms involving optimization calculus are used to achieve the same goals. The research described here involves both of these. The analytical procedure is one proposed by Carroll (1972), and the iterative algorithm is one used in Takane, Young, and deLeeuw's (1977) ALSCAL program. An unfolding technique called Successive Unfolding, which successively uses each procedure, will be described. Use of Successive Unfolding will be illustrated by analyzing some family preference data.

\section{Carroll's External Analysis of Preference Data}

Carroll (1972) defined a linear-quadratic hierarchy of models to do external analysis of preference data. Each of these models involves a regression analysis, in which a Subject $\times$ Stimulus matrix of preference rank orders is regressed on the coordinates and functions of the coordinates of the existing stimulus configuration.

His models can be summarized as follows. Model I, the most general model, gives subjects a great deal of freedom; subjects are assumed to share a common perceptual space (in which the stimulus configuration already exists) but may choose their own set of (orthogonal) reference axes in that space, and then may differentially weight the axes. In addition, negative weights are permitted, so a subject's preference function on any dimension may be single peaked or single dipped. In Model II, subjects may differentially weight dimensions and may have negative weights but are no longer free to select their own reference axes. Carroll suggests that an "average subject" reference axis may be obtained for the Model II common axes from Model I. In Model III all subjects must use the same reference axes and the same weights (and "average subject" values of these can be obtained from Model II analysis), so this model assumes that differences in distances are the same for all subjects. Single 
peaked and dipped preference functions also exist here. Model IV, on the other hand, assumes monotonicity of preferences on all dimensions and is called the vector model.

As emphasis of the importance of Carroll's development, it should be noted that internal unfolding, the simultaneous scaling of subject and stimulus points, has repeatedly provided computational problems for its users. Specifically, degenerate solutions in which all stimulus points collapse into one cluster and all subject points into another are frequently obtained (see Carroll \& Arabie, 1980, pp. 625-626, for an explanation of this degeneracy). Carroll's external unfolding solution avoids this problem.

\section{ALSCAL}

ALSCAL is a general individual differences multidimensional scaling program developed by Takane, Young, and deLeeuw (1977). It estimates a starting configuration, then iteratively alternates between optimally scaling the data and estimating the model parameters, a procedure they call Alternating Least Squares. As such, it can handle metric or nonmetric data (in addition to missing data) generated by either a discrete or a continuous measurement process and existing at various measurement levels. Two of ALSCAL's characteristics will be of principal interest here. First, the user has the option to input the starting stimulus configuration. Second, the user may specify to the program that the initial stimulus configuration is not to be modified (i.e., it is "fixed" at the initial values).

The Successive Unfolding to be discussed here involves four steps:

1. Computing a matrix of inter-stimulus distances;

2. Using metric ALSCAL to obtain a stimulus configuration from the matrix of distances (a Simple Euclidean MDS);

3. Using Carroll's regression procedure to solve for subject "ideal points";
4. Using this solution as the starting configuration in ALSCAL to do a nonmetric scaling of the preferences into the derived stimulus space.

This approach looks promising enough that further investigation is ongoing, and it may eventually be available as an option in ALSCAL.

\section{Method and Procedure}

Data were collected from a group of University of North Carolina at Chapel Hill undergraduates who were enrolled in an introductory psychology class and who signed up for an experiment on family planning attitudes. In a classroom setting, questionnaires were distributed and filled out under supervision. The data pertinent to this study were obtained by asking the students to rank order their preferences for 18 family types, which are exhibited in Table 1. Students were asked to study a list similar to that in Table 1. Next, they were instructed to "decide which of all the families listed above you would most like to have. Put its number on the line labelled '1st choice,' and cross that family type off the list." This procedure was followed 18 times until a complete rank ordering (Coombs, 1964) was obtained. All students completed the task without difficulty. In addition to the rank ordering, demographic data were also collected so that the students could be classified by sex, race, and religion.

From these responses a Subject $\times$ Family Type $(N \times 18)$ matrix was constructed. Next, distances between stimuli (family types) were computed using the Euclidean distance formula (the square root of the sum of squared differences between pairs of columns). This produced an 18 $\times 18$ symmetric matrix of distances between stimuli. Euclidean distances were used in this step because they are most appropriate to the ALSCAL analysis. Using ALSCAL with such distances is equivalent to performing a principal components analysis on the preference data. 
Table 1

The 18 Family Types Used in This Analysis

\begin{tabular}{ll}
\hline Abbreviation & Description \\
\hline 0 & No children \\
B & One boy \\
G & One girl \\
BB & Two boys \\
GG & Two girls \\
BG & A boy then a girl \\
GB & A girl then a boy \\
3B & Three boys \\
3G & Three girls \\
BGG & A boy then two girls \\
GBG & A girl then a boy then a girl \\
GGB & Two girls then a boy \\
GBB & A girl then two boys \\
BGB & A boy then a girl then a boy \\
BBG & Two boys then a girl \\
$4+=$ & Four or more, equal boys and girls \\
$4+B$ & Four or more, more boys than girls \\
$4+g$ & Four or more, more girls than boys \\
\hline
\end{tabular}

This distance matrix was metrically scaled using ALSCAL in three, two, and one dimensions. The three-dimensional solution, shown in Figure 1, was chosen as most appropriate due to ease of interpretation and goodness of fit $(R-$ Square $=.99) . R-$ Square can be interpreted as the proportion of variance in the original distances accounted for by the final configuration. (Note that in $K-1=17$ dimensions for metric scaling or $K-2=16$ dimensions for nonmetric scaling, a perfect fit is assured; see Lingoes, 1971. In this case, the first three dimensions accounted for virtually all of the variance.) This three-dimensional solution was the stable stimulus configuration used in the external unfolding analysis.

With the stimulus configuration defined, Carroll's regression procedure was used to do an external unfolding. Model III was chosen for this analysis, for several reasons. First, Model IV was considered inappropriate because most subjects clearly have a nonmonotonic preference func- tion for family size. Coombs (1976) found that across five cultures family preference data from $85 \%$ to $90 \%$ of the subjects she studied could be fit with an unfolding model. Modeis I and II would have been appropriate in this case but would have precluded easy comparison across subjects, since these models permit more individual freedom for defining and weighting directions in the stimulus space. Furthermore, the authors felt that for illustrating Successive Unfolding, the simplest unfolding model would best serve the purposes of this study. A discussion of model comparisons in this phase will be presented later.

Subjects' ideal points were determined in the following manner. The regression for Model III involved the equation

$$
P_{i j}=a x^{* 2}+\sum_{t} b_{t} x_{t}+c
$$


where

$P_{i j}$ is the preference of subject $i$ for stimulus $j$;

$X_{t}$ is a variable containing the stimulus coordinate on the $t^{\text {th }}$ dimension;

$X^{* 2}$ is a variable containing the sum of squares of the coordinates; and

$a, b$, and $c$ are regression coefficients.

The predictors in this case were the stimulus coordinates on each of the three dimensions and a fourth "dummy" variable, the sum of squares of the coordinates. Given the regression solution, the coordinates of the ideal point can be solved for using the equation

$y_{i t}=-\frac{1}{2}\left(\frac{b_{i t}}{a_{i}}\right)$

where $y_{i t}$ is the coordinate for subject $i$ on the $t^{t h}$ dimension. Even though Carroll's development permits both "ideal" and "anti-ideal" points for Model III, Equation 2 implies that subjects have a single peaked (but not dipped) preference function for the various dimensions underlying family types. (Intuitively, it seems rather unlikely that an individual would like small and large families, but not medium-sized ones, and so at least for family sizes, this assumption seems quite reasonable.)

An interesting characteristic of this approach is that although the whole preference rank-order matrix can be used in the regression, solving the equation for all subjects simultaneously is equivalent to solving it for them one by one. Practically, what this means is that new subjects can be scaled as time and convenience permit.

With the regression solution defined, the configuration of ideal points was submitted as the starting configuration to ALSCAL. The derived stimulus configuration of family types (Figure 1) was input also, and the program options were used to fix the configuration of family types in the space.' The data were the preference rank

${ }^{1}$ The ALSCAL option to achieve this is implemented by specifying to "read, print, and fix initial column stimulus configuration" in column 24 of the INPUT/OUTPUT OPTIONS card. Specifying to "read and print initial stimulus configuration" in column 20 of this same card informs the program that the starting configuration will be input. orders. ALSCAL iterated nonmetrically until it achieved its exit criterion, at which point the fit of the spatial model to the preferences was considered optimal.

By classifying subject ideal points on demographic characteristics, subgroup differences can be observed. In these external unfolding analyses, the sex and race of each subject were known. By scaling males and females and blacks and whites, differences between these groups in defining family preferences were discernible.

\section{Results}

As described, prior to the external unfolding a Simple Euclidean MDS was obtained from the preference-derived distances between the 18 family types. Three directions in this three-dimensional space are interpretable. Figure 2 shows exactly the same space as Figure 1, but the viewpoint of the space is controlled so that these directions may be seen. The horizontal dimension in Figure 2 can be considered a familysize dimension. On the far right is a cluster of small (one- and two-child) families, in the middle are medium-sized (three-child) families, and on the left are large (four- or more child) families. The point for zero-child families is in the front of the space in Figure 2 and is separate from the other family types. The vertical dimension in Figure 2 is a sex preference dimension. Except for zero-child families and four-or more child families with equal boys and girls, each family type can be considered either a boybiased or a girl-biased family. When there are more of one sex than the other, the bias is for the more numerous sex (e.g., GGB is a girl bias). When there are an equal number of each sex, order is considered, with the first child dictating the preference (e.g., BG is boy biased). The family types almost perfectly divide in half on this distinction, the boy-biased family points in the bottom half and the girl-biased families in the top half of the space. (The one exception was the GBB family, which was scaled into the girl preference side.) The dimension looking into the space in Figure 2 can be interpreted as a balance 


\section{Figure 1}

Overall Stimulus (Family Type) Space in Three Dimensions

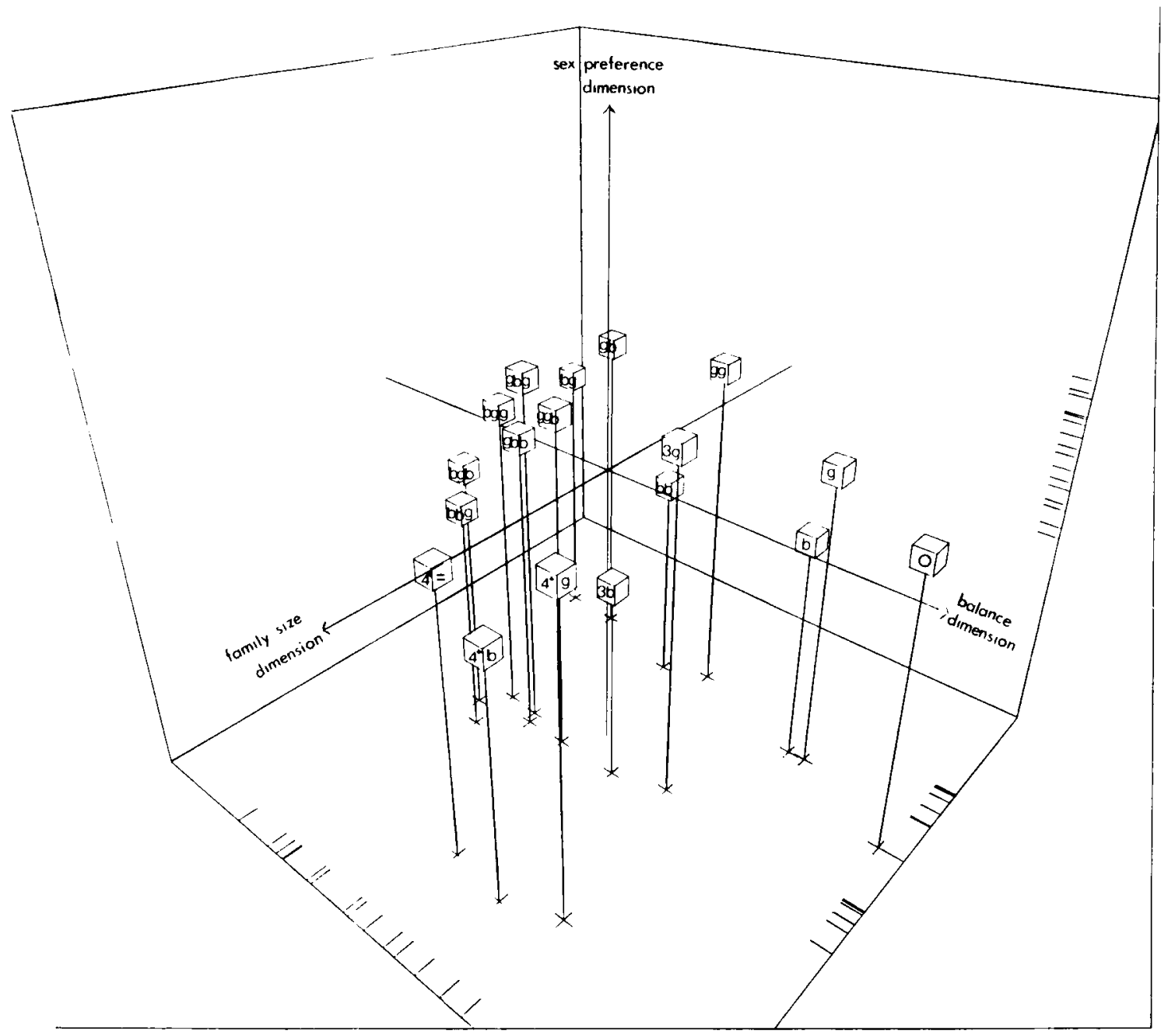

dimension. In the back half are families with both boys and girls (heterogeneous families), and in the front half are families with only boys or only girls (homogeneous families).

Using the Carroll-ALSCAL technique, subject points were scaled into this space. Four different scalings will be reported-for males, females, blacks, and whites; 84 females, 79 males, 80 whites, and 18 blacks were scaled. (These included all of the males and blacks from the original sample of 222 subjects. A random subset of females and whites were chosen for this scaling.) Computational limitations in ALSCAL restricted the number of subjects to around 85; and although multiple runs could have scaled more subjects, these sample sizes were considered adequate for showing group differences.

The external unfoldings are shown by sex and by race in Figures 3 and 4, respectively. For ease of comparison, two 2-dimensional plots (rather than one 3-dimensional one) are shown for each space. The boxes correspond to the family types 
Figure 2

Overall Stimulus Space with View of Interpretable Dimensions

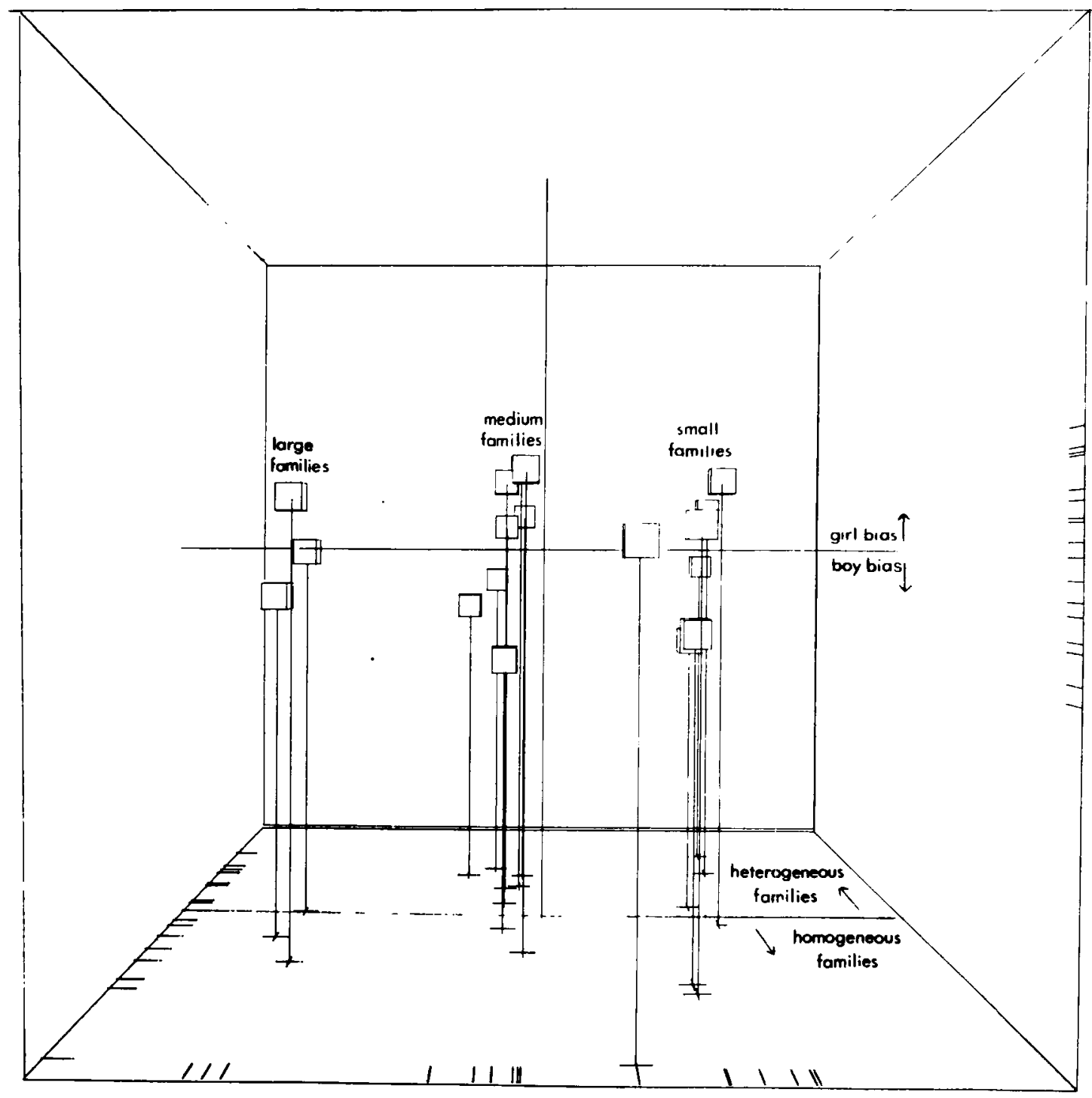

(shown in Figures 1 and 2) and the small circles to subject ideal points. The lines divide the space on the three interpretable dimensions, and the divisions are identified with abbreviations of the divisions shown in Figure 2.

Comparison across subgroups was done by observing the proportions falling in each division. These proportions are shown in Table 2. Pref- erences on these factors can thus be summarized as follows. Males had a slightly stronger boy bias and slightly weaker heterogeneous family bias than females. Both desired small families, and this desire was stronger for males than for females. Blacks showed a markedly stronger boy bias than whites, as well as a stronger small family bias. Interestingly, a majority of blacks 


\section{Figure 3}

Successive Unfolding by Sex

(a) Sex Preference by Family Size Dimensions for Females

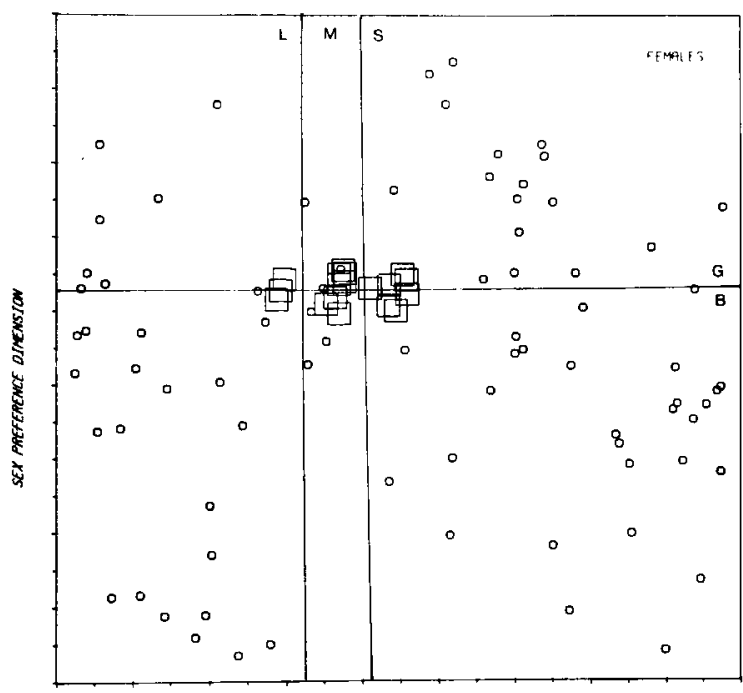

FAMILY SIZE DIMENSION

(c) Sex Preference by Family Size Dimensions for Males

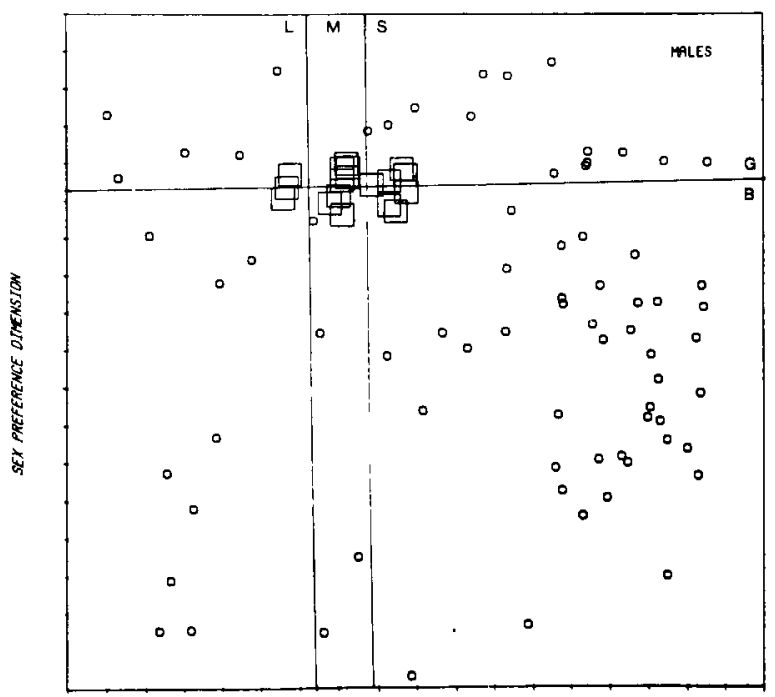

FAMILY SIZE OIMENSION (b) Balance by Family Size Dimensions for Females

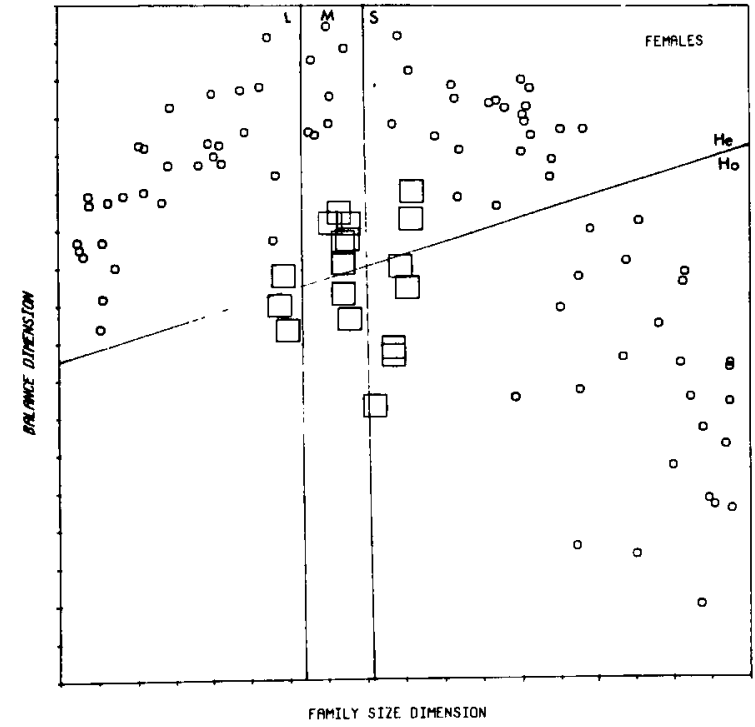

(d) Balance by Family Size Dimensions for Males

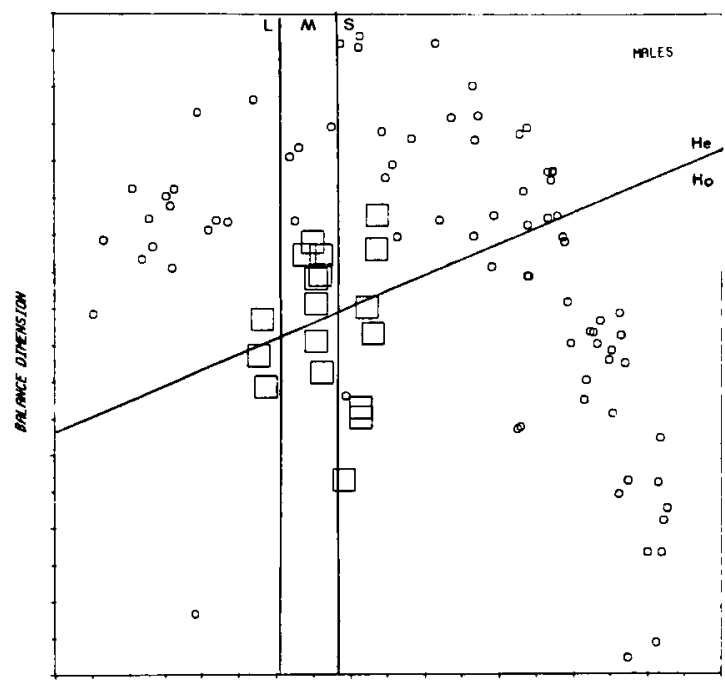

FAMILY SIZE DIFENSION

Downloaded from the Digital Conservancy at the University of Minnesota, http://purl.umn.edu/93227. 


\section{Figure 4}

Successive Unfolding by Race

(a) Sex Preference by Family Size Dimensions for Whites

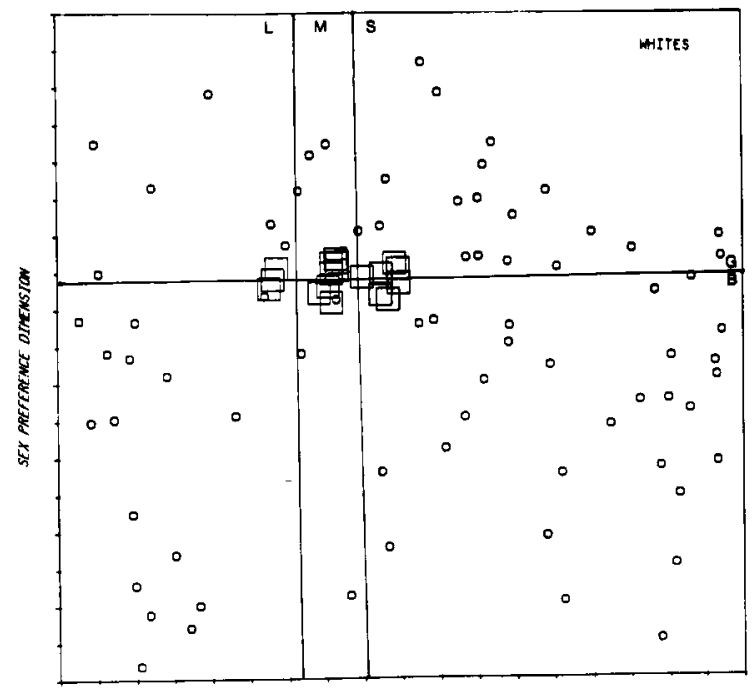

FAMILY SIZE OIMENSION (b) Balance by Family Size Dimensions for Whites

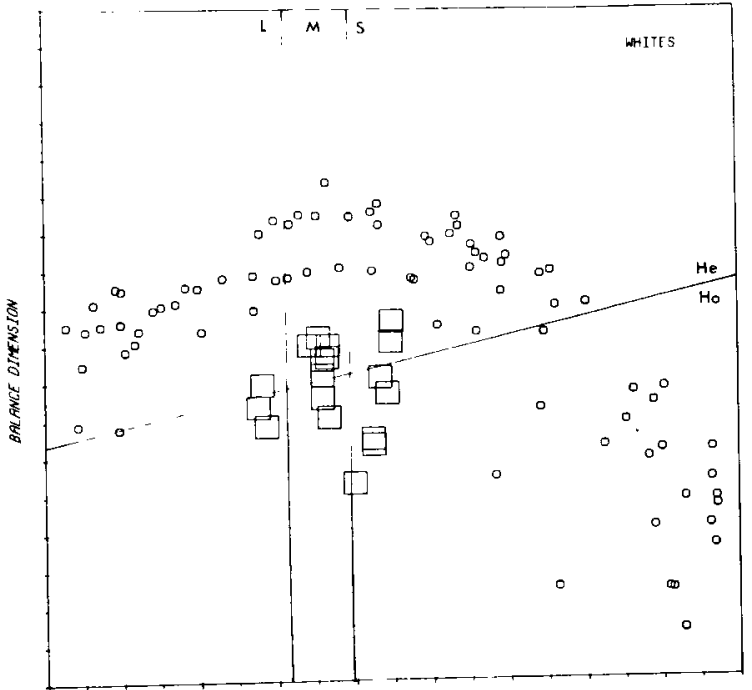

FAMIIY SIZE DIMENSIUN (c) Sex Preference by Family Size Dimensions for Blacks

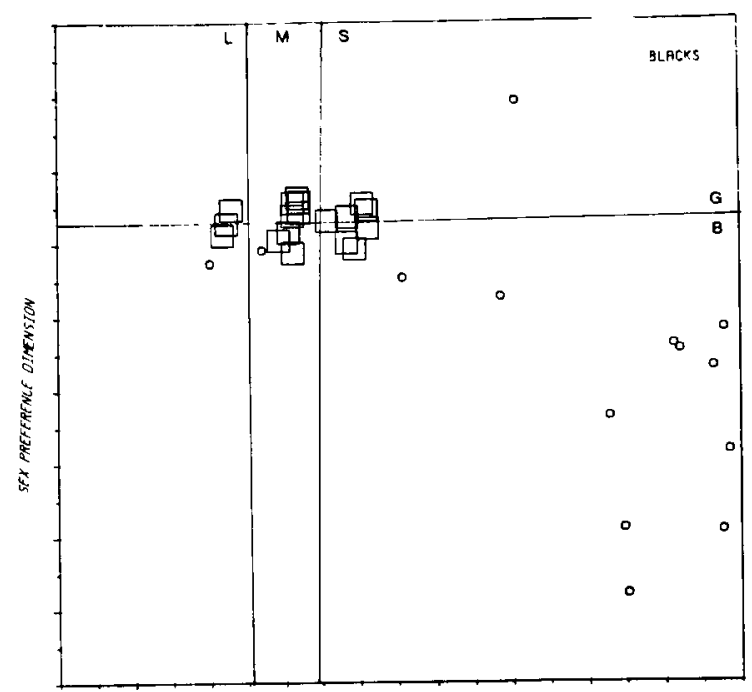

FAMII Y SIZE OIMENSION (d) Balance by Family Size Dimensions for Blacks

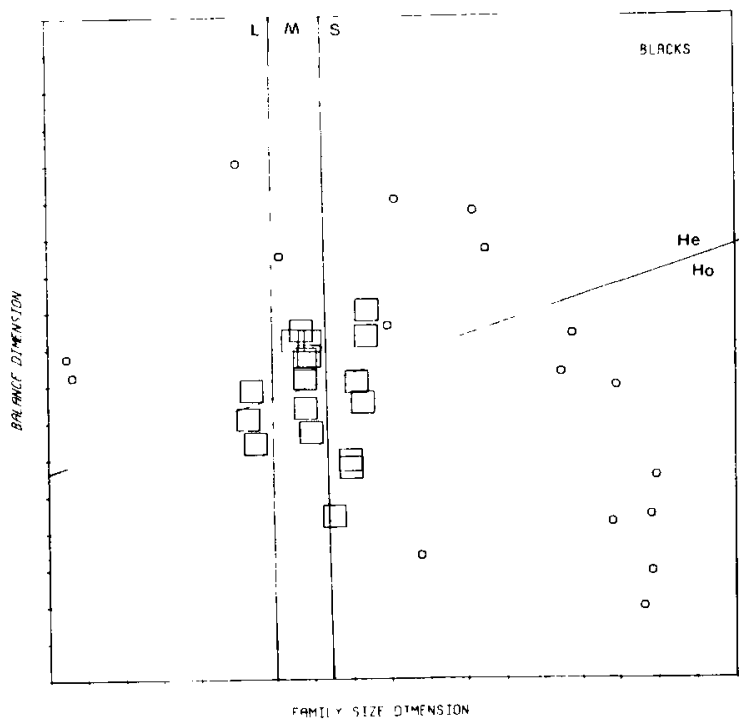


Table 2

Percentages Favoring Family Types by Sex and Race

\begin{tabular}{lcccc} 
& Males & Females & Whites & B1acks \\
\hline Family Size & & & & \\
$\quad$ Large & $21 \%$ & $35 \%$ & $31 \%$ & $18 \%$ \\
Medium & $5 \%$ & $8 \%$ & $9 \%$ & $6 \%$ \\
Smal1 & $74 \%$ & $57 \%$ & $60 \%$ & $76 \%$ \\
Sex Bias & & & & \\
$\quad$ Boy Bias & $74 \%$ & $67 \%$ & $73 \%$ & $93 \%$ \\
$\quad$ Gir1 Bias & $26 \%$ & $33 \%$ & $27 \%$ & $7 \%$ \\
Balance Bias & & & & $47 \%$ \\
$\quad$ Heterogeneous Family & $64 \%$ & $70 \%$ & $73 \%$ & $43 \%$ \\
$\quad$ Homogeneous Family & $36 \%$ & $30 \%$ & $27 \%$ & $53 \%$ \\
\hline
\end{tabular}

preferred homogeneous families (influenced, certainly, by the degree of their small family bias, in which balance is not nearly as relevant as in larger families). The small family preference among blacks is not an unusual result (see Mendoza, 1967), contrary to what many believe. The small sample size of blacks made this group less than ideal for inferential purposes, however, and precluded investigation of Race $\times$ Sex interactions. Whites maintained the expected heterogeneous family bias.

\section{Discussion}

This technique has revealed three substantive results. First, the attributes underlying the way these subjects chose their family preferences have been illuminated in the stimulus space derived in the Euclidean MDS. Second, ideal points for individual subjects have been obtained in relation to dimensions representing these attributes by using external unfolding. And finally, groups of ideal points have been compared to show group differences on these factors.

The use of Carroll's regression in combination with ALSCAL is an advantageous union for both components. ALSCAL is, under certain circumstances, subject to local minimum problems (especially in unfolding), due to its initial con- figuration estimation procedure. Using ALSCAL alone to unfold the females in this study resulted in problems of this nature. Carroll's regression procedure often provides adequate solutions, but in several cases ALSCAL has improved the fit of these solutions. In the four sex and race categories presented here, ALSCAL only needed two iterations from the regression solution to achieve its exit criterion. In a sense, then, ALSCAL only "fine tuned" the solutions obtained from the regression analysis in these problems. Regressions of the same subjects broken down by four religious subgroups were not quite as good; in these ALSCAL needed $9,5,3$, and 2 iterations to exit. So in some cases ALSCAL can improve on the initial configuration obtained by external unfolding.

Various other techniques have been used to measure family preferences and the contributing influences. These include parity progression ratios, preferred sex of first child, and preferred family size (see Coombs, 1977, for a review of these). The current state of the art in family preference measurement is represented by the technique described in Coombs, Coombs, and McClelland (1975). This approach involves the use of conjoint measurement analysis to test the fit of conjoint measurement models to a subject's data, with indices of sex and number preferences derived from unidimensional unfolding 
analyses of sex and number preferences. A further elaboration of this technique by McClelland (1979) uses stopping rule measures along with the unfolding scales in analyses that are even more sensitive to underlying preferences.

Thus far, Successive Unfolding has been developed as a purely descriptive technique, depending heavily on careful inspection of the derived spaces in the MDS solutions. On the other hand, the Coombs et al. (1975) technique for assessing family preferences involves testing the fit between specified models and the data, and as such it has model-building and theory-testing capabilities. The authors of this paper believe Successive Unfolding has this potential, also. Its theoretical base must be developed and strengthened, however, and work on this is currently in progress. The thrust of that work will be summarized here, following a comparison of Successive Unfolding and the Coombs et al. (1975) technique.

Successive Unfolding as applied to family preferences has several important relationships to the Coombs et al. (1975) approach. Both are concerned with validating the legitimacy of the components influencing family preference decisions. The two techniques approach this validation from opposite directions, however. Coombs et al. define composition models and test the fit between the models and the data. To the extent that the fit is good, the specified model is appropriate. In Successive Unfolding the stimulus structure is extracted directly from the data and portrayed in the stimulus space. Here, components are "discovered" rather than specified.

The distinction between exploratory and confirmatory data analysis is illuminating here. If the experimenter has little idea about the influences contributing to a subject's preferences, Successive Unfolding can suggest those influences and how a subject's preferences are defined over them. In this case, however, the model specification necessary in the Coombs et al. (1975) approach would be difficult. If, however, the researcher can specify with some degree of confidence the components influencing preferences, the tests of conjoint measurement axioms involved in the Coombs et al. approach provides a great deal of information about each subject (some of which is not available in a Successive Unfolding analysis). The techniques are useful in different (and, in fact, complementary) situations-Successive Unfolding in more preliminary and exploratory phases of a research problem and the Coombs et al. approach in later confirmatory phases.

The Successive Unfolding technique can be considered a model of a decision-making process in the following sense. If it is assumed that there are societal or cultural factors influencing a subject's preferences for a set of stimuli, the stimulus space derived in the second phase of Successive Unfolding can be viewed as a reflection of those influences. On the other hand, specific preferences within the framework of the stimulus structure are defined at an individual level and are represented by the subjects' ideal points. For instance, subjects may realize that there are societal pressures to have a small family and that small families are those with either one or two children. How much influence that realization has will vary across individuals, however.

The apparent circularity built into the Successive Unfolding analysis presented here (subject preferences are used to define a stimulus space into which those same subjects are scaled) can be considered the reflection of these two different levels of preferences. The stimulus space reflects the structure of the general societal preferences as defined by this sample, and it is assumed that subjects recognize and (at least subconsciously) organize a set of stimuli according to these societal norms. In the external unfolding analysis, preferences are defined in relation to this societal framework. Very good fits of the subject ideal points in the stimulus space are to be expected, because these points are scaled into a space they helped define. (Note that this is only true when the stimulus space is derived directly from the data, as in this case. A stimulus space 
defined by the researcher or derived from different data would not have this characteristic.) Scaling subjects into this space is not a problem interpretationally, since different subjects should have different preferences in relation to this space. The technique must be accompanied by a warning, however, concerning the interpretation of good fits of ideal points. Most, and perhaps all, of the ideal points must fit well, for the reason mentioned above.

Tests of the Successive Unfolding model would involve two phases. First, the goodness of fit of the stimulus space can be considered an index of how well the cultural norms as perceived by this sample are captured in the stimulus space; and the appropriate preference function for an individual across this stimulus space can be obtained by testing the fit of each of Carroll's four models. Tests of these models are not well defined, however, and may have some statistical problems associated with them. This is being studied.

\section{Conclusion}

A Successive Unfolding solution can be produced using Carroll's (1972) regression procedure with Takane, Young, and deLeeuw's (1977) ALSCAL. The solutions were quite interpretable and provided useful substantive results. Furthermore, the technique seems general enough to suggest that other preference data of this type could be so scaled in relation to an externally derived stimulus configuration. Finally, Successive Unfolding can be considered to model a decision-making process occurring in the context of cultural norms, and tests of the model may be developed in the future.

\section{References}

Carroll, J. D. Individual differences and multidimensional scaling. In R. Shephard, A. Romney, \& S. Nerlove (Eds.), Multidimensional scaling: Theory and applications in the behavioral sciences Vol. I, Theory. New York: Seminar Press, 1972.
Carroll, J. D., \& Arabie, C. P. Multidimensional scaling. Annual Review of Psychology. 1980, 31, 607-649.

Coombs, C. H. Theory of data. New York: John Wiley \& Sons, 1964.

Coombs, C. H. Psychological scaling without a unit of measurement. Psychological Review, 1950, 57, 145-158.

Coombs, C., Coombs, L. C., \& McClelland, G. Preference scales for number and sex of children. Population Studies, 1975, 29, 273-298.

Coombs, L. C. Are cross-cultural preference comparisons possible? A measurement theoretic approach (IUSSP Paper No. 5). Leige, 1976.

Coombs, L. C. Preference for sex of children among U.S. couples. Family Planning Perspectives, 1977, 9, 259-265.

Lingoes, J. C. Some boundary conditions for a monotone analysis of symmetric matrices. Psychometrika, 1971,36, 195-203.

McClelland, G. H. Determining the impact of sex preferences on fertility: A consideration of parity progression ratio, dominance, and stopping rule measures. Demography, 1979, 16, 377-388.

Mendoza, E. Social-economic correlates of attitudes toward family size. In E. J. Bogue (Ed.), Mass communication and motivation for birth control. Chicago: University of Chicago Press, 1967.

Takane, Y., Young, F., \& deLeeuw, J. Nonmetric individual differences multidimensional scaling: An alternating least squares method with optimal scaling features. Psychometrika, 1977, 42, 7-67.

Torgerson, W. S. Theory and method of scaling. New York: John Wiley \& Sons, 1958.

\section{Acknowledgments}

The authors wish to thank Mark Appelbaum, Vaida Thompson, and David Budescu of the University of North Carolina at Chapel Hill, Psychology Department, and two anonymous reviewers for their insightful comments. This research was completed as part of the first author's master's thesis, and was partially supported by NICHD Grant 5T01-HD0019310 and NIMH Grant 1-T32-MH15745-01.

\section{Authors' Address}

Send requests for reprints and further information to Forrest W. Young, The L. L. Thurstone Psychometric Laboratory, Davie Hall 013A, The University of North Carolina at Chapel Hill, Chapel Hill, NC 27514. 